\title{
Estratégias competitivas e de desenvolvimento de produtos lácteos funcionais: estudos de caso em empresas agroindustriais da região Sul do Brasil
}

\author{
Competitive and product development strategies in functional dairy products: case studies in food \\ processing industries from south Brazil
}

\author{
Mateus Silva de Lima ${ }^{\mathrm{I}}$ Jean Philippe Palma Révillion ${ }^{\mathrm{I}^{*}}$ Antonio Domingos Padula
}

\section{RESUMO}

\begin{abstract}
Esta pesquisa buscou compreender o motivo e o modo como as empresas agroindustriais brasileiras desenvolvem novos produtos e se orientam para o mercado no segmento de alimentos lácteos funcionais. Ela também examina o papel do sistema regulatório nesse processo. Foi utilizada a abordagem de estudos de caso para esclarecer as inter-relações sistêmicas e trade-offs entre os fatores relacionados à inovação. Foi escolhido um desenho de pesquisa com uma unidade de análise (empresas agroindustriais que, recentemente, lançaram novos produtos no mercado de lácteos funcionais) e estudos de caso múltiplos (três empresas agroindustriais da região $\mathrm{Sul}$ ). Os resultados revelam que as empresas agroindustriais adotam estratégias seguidoras em relação às concorrentes multinacionais. Tradicionalmente, essa é uma estratégia considerada mais segura e exige menores investimentos em $P \& D$ e marketing. Contudo, ela corrói a imagem de marca e limita os retornos financeiros das inovações. O comportamento seguidor é resultado de um baixo grau de orientação para mercado e restrita cooperação com parceiros externos. Esse "ciclo vicioso" é reforçado pelas barreiras estabelecidas pelo sistema regulatório.
\end{abstract}

Palavras-chave:

estratégias seguidoras, empresas agroindustriais, inovação, alimentos lácteos funcionais.

\section{ABSTRACT}

This multi-case study searched to understand why and how food processing industries develop new products and market orientates to compete in the Brazilian functional dairy market. It also examines the regulatory framework role in this process. We use case studies as an approach to give evidence of the systemic relations and the existent trade-offs among factors related to innovation. We chose a research design with one unity of analysis (food processors that recently launched new products in the functional dairy market) and multiple case studies (three Brazilian dairies of the southern region). The results reveal that the enterprises adopt a second-mover strategy vis-à-vis the multinational competitors. Traditionally, this strategy is considered safer and requires lower $R \& D$ and marketing investments. However, it erodes branding and also limits financial returns from innovations. The second-mover behavior is consequence of poor market orientation and restricted cooperation with external partners. This "vicious circle" is also enhanced by barriers established by the regulatory framework.

Key words: second-movers, food processing firm, innovation, functional dairy foods.

\section{INTRODUÇÃO}

Segundo o European Functional Food Science Programme, alimento funcional é aquele que permite demonstrar, de forma satisfatória, uma relação entre seu consumo e um efeito benéfico em uma ou mais funções do organismo humano, além dos benefícios nutricionais, melhorando o estado de saúde e bemestar e/ou reduzindo o risco de doenças para o consumidor (DIPLOCK, 1999).

Nos principais países industrializados, os alimentos funcionais representam a categoria em que se concentra a maior parte das inovações do setor de alimentos, pois, nesse mercado, os lácteos funcionais

'Programa de Pós-graduação em Agronegócios, Centro de Estudos e Pesquisas em Agronegócios (CEPAN), Universidade Federal do Rio Grande do Sul (UFRGS), Rua Washington Luis, 855, 90010-460, Porto Alegre, RS, Brasil E-mail: jeanppr@gmail.com.

*Autor para correspondência. 
respondem por quase $65 \%$ das vendas (MENRAD, 2003). É importante destacar que a categoria de produtos fermentados, com características probióticas, são a grande maioria. Esses produtos têm, em sua composição, microrganismos que permanecem vivos no trato gastrointestinal, possuindo alegação de saúde que associa seu consumo a diversos benefícios à saúde, dentre eles, melhor funcionamento digestivo e prevenção de certos tipos de câncer.

O conhecimento sobre o comportamento do consumidor de alimentos funcionais é, contudo, incipiente (HOOLIHAN, 2003) e varia entre diferentes países em função das particularidades regionais (LÄHTEENMÄKI, 2003).

Além disso, os alimentos funcionais apresentam atributos de “credencial”, ou seja, características que não podem ser comprovados pelo consumidor nem antes nem após a compra: o consumidor precisa confiar na informação transmitida em certificados, na embalagem, pela mídia e outras formas de comunicação (DARBY \& KARNI, 1973) um processo complexo que depende tanto da credibilidade da fonte como da capacidade do consumidor em perceber esses indicadores (GRUNERT et al., 1997).

Esta pesquisa pretende contribuir com a compreensão de por que e como as empresas agroindustriais brasileiras desenvolvem produtos lácteos funcionais e comunicam suas particularidades, para atender novas necessidades dos consumidores, visando a ampliar sua presença no mercado. Também são avaliadas as restrições e estímulos emergentes do sistema institucional relacionados ao processo de inovação dessa categoria de produtos.

Essa análise é especialmente pertinente, considerando-se que as empresas de pequeno e médio porte do setor processador de laticínios tendem a adotar estratégias seguidoras diante de algumas desvantagens competitivas em relação às empresas de maior porte de atuação multinacional: como menor capacidade de consolidar a marca e desenvolver estratégias de diferenciação e, também, deseconomias de escala e escopo (DIRVEN, 2001; RÉVILLION, 2004).

De fato, existem vantagens e desvantagens, decorrentes da escolha do momento de entrada em um mercado, que devem ser consideradas (GILBERT \& BIRNBAUM-MORE 1996; MUELLER, 1997) para se ponderar a sustentabilidade das estratégias competitivas desenvolvidas pelas empresas agroindustriais nesse setor.

\section{MATERIAL E MÉTODOS}

O método utilizado foi o estudo de caso (YIN, 1994) de três empresas agroindustriais brasileiras inovadoras no mercado de lácteos funcionais e construído a partir de levantamentos de dados em fontes secundárias (publicações científicas, páginas especializadas da Internet e periódicos), realização de entrevistas semiestruturadas com stakeholders, balizadas por questões relacionadas ao referencial teórico pertinente à inovação no mercado de alimentos funcionais (disponível sob demanda aos autores).

A triangulação dos dados obtidos a partir de fontes múltiplas: vários tomadores de decisão das organizações analisadas (diretores, gerentes de produção e marketing, responsáveis pelo controle de qualidade), assim como representantes de instituições relacionadas (órgão de defesa dos consumidores PROCON; fornecedores de ingredientes - Chr. Hansen Brazil e B. U. Sweeteners Danisco e; órgãos públicos de fiscalização e normatização) - foi fundamental para a validação das conclusões dos estudos de caso (WESTGREN \& ZERING 1998).

A análise interna dos casos foi realizada de maneira a gerar insights no confronto com a teoria sobre inovação no setor agroindustrial. Já a análise entre casos foi conduzida a fim de evidenciar convergências e divergências na dinâmica do fenômeno estudado. As entrevistas foram realizadas no segundo semestre de 2006 e no mês de janeiro de 2007. Os registros foram feitos por gravação de áudio, todos com o consentimento dos entrevistados.

\section{RESULTADOS E DISCUSSÃO}

$\mathrm{Na}$ tabela 1, são sintetizadas as características do processo de inovação, empreendido nas empresas agroindustriais, objeto dos estudos de caso, no mercado de lácteos funcionais.

Todas as empresas estudadas consideram o mercado de alimentos funcionais uma oportunidade para aumentar sua competitividade e reconhecem que o perfil do consumidor brasileiro, cada vez mais, busca alimentos saudáveis e de maior valor agregado. Esses dados alinham-se ao comportamento observado nos consumidores em nível mundial (ROBERFROID, 1998; LÄHTEENMÄKI, 2003).

Apesar disso, atualmente, elas têm se posicionado como organizações seguidoras, desenvolvendo produtos similares aos que já foram lançados por empresas multinacionais, o que configura uma tendência no setor processador de laticínios (RÉVILLION, 2004; DIRVEN, 2001).

Essa dinâmica já foi observada por GRUNERT et al. (1997) em outros mercados de produtos agroindustriais onde a difusão dos padrões de identidade próprios, por parte das empresas com maior 
Tabela 1 - Características do processo de inovação empreendido nas agroindústrias objeto dos estudos de caso, no mercado de lácteos funcionais.

\begin{tabular}{|c|c|c|c|c|}
\hline \multirow{3}{*}{$\begin{array}{l}\text { Categorias de produtos } \\
\text { funcionais: }\end{array}$} & Organização A & \multirow{2}{*}{$\begin{array}{c}\text { Organização B } \\
\text { Leites fermentados e queijos. }\end{array}$} & \multirow{2}{*}{\multicolumn{2}{|c|}{$\begin{array}{r}\text { Organização C } \\
\text { Leites fermentados. }\end{array}$}} \\
\hline & $\begin{array}{l}\text { Leites especiais e leites } \\
\text { fermentados. }\end{array}$ & & & \\
\hline & Pesquisa com consumidores. & $\begin{array}{l}\text { Contato com colegas de } \\
\text { outras organizações. }\end{array}$ & $\begin{array}{l}\text { Observação direta } \\
\text { pontos de venda. }\end{array}$ & nos \\
\hline
\end{tabular}

Ferramentas de identificação de oportunidades de mercado:

Motivações para inovar:

Estratégia competitiva:

Sistemas de

desenvolvimento de novos produtos:

Particularidades da estratégia de comunicação:

Papel do sistema

institucional:
Observação da concorrência e retorno do “sistema de apoio aos clientes”.

Ampliar a linha de produtos e agregar valor à imagem da organização.

Seguidora (com inovações incrementais) para incorrer em menores investimentos de $P \& D$, comunicação e registro de novos produtos.

Não utiliza ferramentas ou sistemáticas específicas, ênfase no controle de qualidade dos processos e produtos. Integração parcial entre setores de produção e marketing. Importante subsídio dos fornecedores de ingredientes.

Linha específica e característica com padrões semelhantes à concorrência. Estratégias de promoção voltadas a profissionais da área de saúde e alimentação.

Reconhecimento de avanços importantes na legislação sobre alimentos funcionais. A fiscalização é considerada fundamental para barrar ações oportunistas, porém é considerada pouco eficiente. capacidade de investimentos em comunicação e consolidação de marca, gerou barreiras para as empresas seguidoras desenvolverem e comunicarem atributos diferenciais de seus produtos.

De fato, os investimentos em comunicação são críticos no mercado de alimentos funcionais, considerando a necessidade de uma alta freqüência de consumo para que seus benefícios sejam perceptíveis, os quais proporcionarão a fidelização dos consumidores (LÄHTEENMÄKI, 2003). Além disso, é fundamental desenvolver esforços de promoção e relações públicas voltados aos profissionais da saúde “formadores de opinião” (MENRAD et al., 2000). São iniciativas cuja importância é percebida pelas organizações estudadas.

Também foi constatado, em todas as empresas agroindustriais pesquisadas, que o lançamento dos alimentos funcionais foi realizado em uma linha específica e característica (com um padrão de embalagem diferenciado, embora semelhante ao das empresas pioneiras). A ampliação das linhas de produtos ofertados é fundamental para incrementar as possibilidades de acesso às grandes redes varejistas e, ao mesmo tempo, consolidar a imagem da marca junto ao consumidor final - como observado por RÉVILLION (2004) para outros produtos lácteos.

Com relação às fontes de inovação, foi possível identificar o importante papel desempenhado pelos fornecedores de ingredientes no desenvolvimento das inovações empreendidas nas empresas pesquisadas. De fato, os investimentos em
P\&D interno não são prioritários no setor agroindustrial e a inovação é dependente de fornecedores de equipamentos e insumos (CHRISTENSEN et al., 1996).

Tanto os trade-offs, relacionados à adoção de estratégias pioneiras ou seguidoras, como a importância das capacitações da organização para implementar essas estratégias, ficam evidenciados ao se considerarem as estratégias competitivas, relacionadas à principal categoria de produtos funcionais desenvolvida pelas empresas consideradas nos estudos de caso: os leites fermentados.

Por exemplo, a empresa “ $A$ ” foi pioneira no Brasil, em meados da década de 1990, na oferta de leites fermentados com características funcionais, porém ela não conseguiu acessar ou desenvolver esse mercado satisfatoriamente por não possuir uma rede de distribuição adequada e/ou ações de publicidade e promoção consistentes para alavancar o consumo desses produtos inovadores. De fato, somente em 2004, com o lançamento da linha Activia, pela Danone, é que essa categoria de produtos se tornou menos "exótica” para o consumidor, através de maciços investimentos em comunicação.

De outro lado, as empresas estudadas que adotaram estratégias seguidoras (" $B$ ” e “C”) não desenvolveram uma linha de leites fermentados com diferenciais significativos em relação aos produtos estabelecidos no mercado. Nessas organizações, mesmo com a possibilidade de acessar fornecedores de ingredientes e coadjuvantes de tecnologia inovadores, para se desenvolverem produtos inéditos 
voltados a nichos específicos de consumidores, seria previsível a rápida resposta da concorrência diante da baixa apropriabilidade dessa estratégia tecnológica (CHRISTENSEN et al. 1996; MARTINEZ; BURNS, 1999).

Nesse contexto, é compreensível a predominância de estratégias seguidoras e o desenvolvimento de inovações incrementais nas empresas estudadas. Como enfatizaram GILBERT \& BIRNBAUM-MORE (1996), essa posição é de menor risco pela possibilidade de se tomarem decisões em uma situação de menor incerteza tecnológica, mercadológica e regulatória, reduzindo os riscos de investir recursos em $\mathrm{P} \& \mathrm{D}$ e marketing em mercados que se revelam pouco promissores do que aqueles das empresas pioneiras.

Em relação ao papel do sistema institucional, os tomadores de decisão, nas empresas agroindustriais estudadas, consideram que a legislação específica do mercado de alimentos funcionais é dinâmica e necessária para barrar ações oportunistas (convergindo com BECH-LARSEN et al., 2001) e favorecer a confiança do consumidor nesses produtos diferenciados (como observou HOOLIHAN, 2003), porém a fiscalização é insuficiente.

Em especial, o tempo e os custos relacionados ao registro dos novos produtos funcionais são considerados elevados, situação também observada na Finlândia por BISTRÖM \& NORDSTRÖM(2002).

\section{CONCLUSÃO}

A adoção de estratégias competitivas seguidoras pelas empresas agroindustriais estudadas é motivada pela perspectiva de incorrer em menores investimentos em $\mathrm{P} \& \mathrm{D}$ e comunicação no mercado de lácteos funcionais.

Essa estratégia, porém, demonstra a desconsideração de certos aspectos estratégicos relacionados ao ciclo de vida dos produtos: o nível de lucratividade relacionado ao lançamento de novos produtos diminui, à medida que novos concorrentes lançam produtos substitutos ou que as empresas pioneiras desenvolvem estratégias promocionais, deslocando a base competitiva para fatores relacionados à escala de produção e ao grau de acesso ao mercado, que não são características diferenciais das empresas estudadas.

Complementarmente, a restrição de esforços e investimentos relacionados à orientação para o mercado limita o desenvolvimento de capacitações associadas, tanto à detecção e caracterização de novos segmentos de consumidores, quanto ao desenvolvimento de novos produtos diferenciados. Essa realidade se reflete na pouca integração entre os setores de produção (ou P\&D quando existem) e marketing nas agroindústrias estudadas. Além disso, cria-se a percepção de que a inovação radical de produto não é uma meta acessível às empresas domésticas.

Esse comportamento estratégico é reforçado pelas características do mercado de alimentos funcionais em que a inovação depende de investimentos relevantes. Esse dispêndio de recursos está relacionado tanto à realização de experimentos ou estudos de caráter nutricional, capazes de garantir o reconhecimento de sua funcionalidade nos órgãos de regulação, bem como na consolidação de ações de comunicação visando a esclarecer e a educar o consumidor das vantagens decorrentes do consumo desses novos produtos.

As estratégias alternativas, diante desse quadro competitivo, envolvem o desenvolvimento de esforços de cooperação das empresas processadoras de alimentos funcionais com instituições setoriais, ou mesmo instituições de ensino e pesquisa, de maneira a compartilhar os custos e as competências necessários à inovação nesse promissor, mas concorrido mercado.

\section{AGRADECIMENTOS}

Os autores agradecem à Coordenação de Aperfeiçoamento de Pessoal de Nível Superior (CAPES), pelo financiamento deste estudo.

\section{REFERÊNCIAS}

BECH-LARSEN, T. et al. The acceptance of functional foods in Denmark, Finland and the United States. Aarhus, Dinamarca: MAPP, 2001. (working paper, n.73). Disponível em: <http://130.226.203.239/pub/mapp/wp/wp73.pdf $>$. On line. Acesso em: 12 jan. 2009.

BISTRÖM, M.; NORDSTRÖM, K. Identification of key success factors of functional dairy foods product development. Trends in Food Science \& Technology, Cambridge, n.13, p.372379, 2002. Disponível em: <http://www.sciencedirect.com/ science?_ob=ArticleURL\&_udi=B6VHY-4810GS1$9 \&$ \&user $=10 \&$ \&_doc $=1 \&$ \&mt $=$ \&_orig $=$ search \&_sort $=$ d \&vie $\mathrm{w}=\mathrm{c} \&$ \& a c c t $=$ C $000050221 \&$ \& version $=1 \&$ _url version $=0 \&$ _ us erid $=10 \&$ m d $5=$ e e $27 \mathrm{f} 06 \mathrm{~b}$ d45e432330239095d898e741>. Doi: 10.1016/S09242244(02)00187-5.

CHRISTENSEN, J.L. et al. Innovation in the european food products and beverage industry. Industry studies of innovation using C.I.S. data. Bruxelas: European Commission - EIMS Project 94/111 EIMS, 1996. 146p. (Publication n.35).

Ciência Rural, v.39, n.5, ago, 2009. 
DARBY, M.R.; KARNI, E. Free competition and the optimal amount of fraud. Journal of Law and Economics, Chicago, v.16, n.1, p.67-88, 1973. Disponível em: <http:// www.journals.uchicago.edu/doi/abs/10.1086/ 466756\#masthead>. Doi: 10.1086/466756.

DIPLOCK, A.T. Scientific concepts of functional foods in Europe: consensus document. British Journal of Nutrition, Londres, n.81, p.1-27, 1999. Disponível em: <http:// j o u r n a l s. c a m b r i d g e. o r g/ d o w n l o a d. p h p ? f i l e = \% 2 F B J N \% 2 F B J N 81 01\%2FS000711459900003 3a.pdf\&code=3ffadb19d3520 98390aecae20ceafeb1>. On line. Acesso em: 11 jan. 2009.

DIRVEN, M. Dairy clusters in Latin America in the context of globalization. International Food and Agribusiness Management Review, Texas, v.2, n.3/4, p.301-313, 2001. Disponível em: <http://www.sciencedirect.com/ science?_ob=ArticleURL\&_udi=B6W4N-436W0JK$3 \&$ \&user $=687358 \&$ \&doc $=1 \&$ \& fmt $=\&$ \& orig $=$ search \&_sort $=\mathrm{d} \& \mathrm{v}$ i e $\mathrm{w}_{1}=$ c \&_a c c t $=$ C $000037899 \&$ \& versi on $=1$ \&_u r l V e r s i o n $=0 \&$ \& s e $\bar{r}$ i d $=6873$ $58 \&$ md5 =faf9a7bdf35aa44ad9e139c9736ca09f $>$. Doi: 10.1016/S1096-7508(01)00045-3.

GILBERT, J.T.; BIRNBAUM-MORE, P.H. Innovation timing advantages: from economic theory to strategic application. Journal of Engineering and Technology Management, Amsterdam, v.12, p.245-266, 1996. Disponível em: <http:// w w w. s c i e $n$ c e d i r e c t. c o m / science?_ob=ArticleURL\&_udi=B6VF3-46YJR7C18 \&user $=10$ \&_rdoc $=18$ _fmt $=$ \&_orig $=$ search\&_sort $=$ d \&view $=\mathrm{C}$ \&_a c ct $=$ C $000050221 \&$ \& version $=1 \&$ \&_urlVersio $\mathrm{n}=0$ \&_userid=10\&md5=7e731b5b7d060f21732382a2650f4772>. Doi: 10.1016/0923-4748(95)00017-8.

GRUNERT, K.G. et al. A framework for analysing innovation in the food sector. In: TRAIL, B.; GRUNERT, K.G. Product and process innovation in the food industry. Suffolk: Chapman \& Hall, 1997. Cap.1, p.1-37.

HOOLIHAN, L. The market for functional dairy products: the case of the United States. In: MATTILA-SANDHOLM, T.; SAARELA, M. Functional dairy products. Cambridge: Woodhead, 2003. Cap.17, p.378-389.

LÄHTEENMÄKI, L. Consumers and functional foods. In: MATTILA-SANDHOLM, T.; SAARELA, M. Functiona dairy products. Cambridge: Woodhead, 2003. Cap.15, p.346358 .
MARTINEZ, M.G.; BURNS, J. Sources of technological development in the spanish food and drink industry. A "supplierdominated" industry? Agribusiness, Hoboken, v.15, n.4, p.431-448, 1999. Disponível em: <http:// www3.interscience.wiley.com/journal/66500490/ abstract?CRETRY $=1 \&$ SRETRY $=0>$. Doi: $10.1002 /($ SICI) 1520 6297(199923)15:43.0.CO;2-0

MENRAD, K. Market and marketing of functional food in Europe. Journal of Food Engineering, Essex, v.56, n.2-3, p.181-188, 2003. Disponível em: < http://www.sciencedirect.com/ science?_ob=ArticleURL\&_udi=B6T8J-461XJ1F$2 \&$ _user $=10 \&$ \&_doc $=1 \&$ \&mt $=\&$ \&orig $=$ search\&_sort $=\mathrm{d} \&$ vie $\mathrm{w}=\mathrm{c} \&$ a c c $\mathrm{t}=\mathrm{C} 000050221 \&$ \& e r s i o n $=1$ \& _ u r l V e r s i o n $=0 \&$ \& s e r i d $=10$ \&md5=6c7ddb897fff59191e01da345f8296e1>. Doi: 10.1016/ S0260-8774(02)00247-9

MENRAD, M. et al. Functional food. Programm Technology Assessment-TA, 37. Bern: Schweizerischer Wissenschafts und Technologierat, 2000. 363p.

MUELLER, D.C. First-mover advantages and path dependence. International Journal of Industrial Organization, Amsterdam, v.15, p.827-850, 1997. Disponível em: <http:// w w w. s c i e $n$ c e d i r e c t. c o m / science? ob=ArticleURL\&_udi=B6V8P-3SX1KFP$2 \&$ \&user $=10 \&$ \&_rdoc $=1 \&$ \& fmt $=\&$ \&_orig $=$ search \&_sort $=\mathrm{d} \&$ vi e w $=$ c \& a c c t $=$ C $000050221 \&$ ve r s i on $=1$ \&_u r l Vers i o n $=0$ \&_u s e r i d $=10 \&$ m d 5 $=$ ae7377d885602b0077adcacd0bfebd1e $>$. Doi: 10.1016/S01677187(97)00013-1.

RÉVILliOn, J.P.P. Análise dos sistemas setoriais de inovação das cadeias produtivas de leite fluido na França e no Brasil. 2004. 196f. Tese (Doutorado em Agronegócios) - Curso de Pós-graduação em Agronegócios do Centro de Estudos e Pesquisas em Agronegócios. Universidade Federal do Rio Grande do Sul.

ROBERFROID, M. The science of functional food. In: FORUM ON FUNCTIONAL FOOD, 1998, Strasburgo, França. Anais... Strasburgo: Council of Europe, 1998. p.57-66.

WESTGREN, R.; ZERING, K. Case study research methods for firm and market research. Agribusiness, Hoboken, v.14, n.5, p.415-424, 1998.

YIN, R.K. Case study research: design and methods. London: Sage Publications, 1994 p.181. 